\title{
Prevalence of musculoskeletal pain in association with serum 25-hydroxyvitamin $D$ concentrations in patients with type 2 diabetes mellitus
}

\author{
MOHAMMAD J. ALKHATATBEH ${ }^{1}$, KHALID K. ABDUL-RAZZAK ${ }^{1}$, \\ LUBNA Q. KHASAWNEH ${ }^{1}$ and NESREEN A. SAADEH ${ }^{2}$ \\ ${ }^{1}$ Department of Clinical Pharmacy, Faculty of Pharmacy; ${ }^{2}$ Department of Internal Medicine, \\ Faculty of Medicine, Jordan University of Science and Technology, Irbid 22110, Jordan
}

Received February 7, 2018; Accepted April 25, 2018

DOI: $10.3892 /$ br.2018.1093

\begin{abstract}
The aim of the present study was to investigate the prevalence of musculoskeletal pain in patients with type 2 diabetes mellitus (T2DM) in association with 25-hydroxyvitamin D levels, anxiety, depression and neuropathy. A cross-sectional study was conducted involving a total of 124 T2DM patients. Musculoskeletal pain was determined by self-reporting of painful body sites. Pain intensity was assessed using a scale of 0-10. Anxiety and depression were assessed using the Hospital Anxiety and Depression Scale. Neuropathy was assessed using the PainDETECT questionnaire. The concentration of 25-hydroxyvitamin D was measured using liquid chromatography-tandem mass spectrometry. Fasting blood sugar (FBS) was determined using the hexokinase method and glycated hemoglobin (HbA1c) level was determined using turbidimetric inhibition immunoassay. The neck, lower back and head were reported as the most common painful sites (affected in 60.5, 60.5 and 56.5\% of patients, respectively). Pain in the lower extremities, including the knees, lower legs and feet, was more common compared with pain in the upper extremities. The pain measurements of number of painful sites and pain intensity did not differ significantly among patients with sufficient (>30 ng/ml), insufficient (20-30 ng/ml) and deficient $(<20 \mathrm{ng} / \mathrm{ml})$ vitamin $\mathrm{D}$ levels $(\mathrm{P}>0.05)$. The pain measurements were identified to have no correlation with age, body mass index, FBS, HbA1c level, 25-hydroxyvitamin D concentration, anxiety or depression $(\mathrm{P}>0.05)$. However, the pain measurements were correlated with duration of T2DM and neuropathy score $(\mathrm{P}<0.05)$. Further regression analysis demonstrated that the pain measurements were significantly associated with the neuropathy score $(\mathrm{P}<0.05)$. In conclu-
\end{abstract}

Correspondence to: Dr Mohammad J. Alkhatatbeh, Department of Clinical Pharmacy, Faculty of Pharmacy, Jordan University of Science and Technology, Amman-Ramtha Hwy, Irbid 22110, Jordan E-mail: khatatbeh@just.edu.jo

Key words: vitamin D, neuropathy, diabetes, musculoskeletal pain sion, musculoskeletal pain in patients with T2DM was not associated with 25-hydroxyvitamin D concentration, but was associated with neuropathy score. This may encourage further investigations to assess the etiology of musculoskeletal pain in T2DM, and whether vitamin D supplementation and management of neuropathy would be of value as pain relief treatment.

\section{Introduction}

Type 2 diabetes mellitus (T2DM) is a chronic metabolic abnormality that is characterized by hyperglycemia due to decreased insulin secretion and/or insulin resistance (1). The disease typically starts in middle age and its incidence is associated with certain risk factors, including obesity, hypertension, physical inactivity and family history of T2DM (1). To prevent persistent hyperglycemia, patients with T2DM should adhere to their prescribed pharmacological treatment and recommended lifestyle modifications (2), and regularly monitor their blood glucose measurements (3). Otherwise, uncontrolled T2DM may result in long-term macro- and microvascular complications, including atherosclerosis, retinopathy, nephropathy and neuropathy (4). One of the suggested mechanisms that may be implicated in the development of microvascular complications is the formation of advanced glycation end products (AGEs) (5). Of note, a growing body of evidence suggests that increased levels of AGEs, in addition to persistent hyperglycemia, may predispose patients to stiffness of connective tissues, leading to painful musculoskeletal manifestations (6).

Musculoskeletal pain is common in patients with T2DM, and its occurrence is considered to be multifactorial (7). One of the contributing factors is increased body weight (8). T2DM patients are usually obese, and increased body mass index (BMI) has been identified to be associated with musculoskeletal pain, particularly in the lower extremities (8). In addition, T2DM patients may suffer from symptoms of neuropathy as a complication of the disease itself; these symptoms include numbness, tingling, burning sensation, muscle weakness and pain (9). Musculoskeletal manifestations in patients with T2DM may also result from vitamin D deficiency (10). This vitamin is involved in maintaining calcium and phosphate homeostasis by controlling intestinal absorption of both 
minerals (11). Thus, vitamin D deficiency may cause defective bone mineralization, leading to bone pain, tenderness, muscle weakness and myopathy (12). In addition, vitamin D deficiency has been observed to be associated with the development of anxiety and depression $(13,14)$, which may also cause musculoskeletal pain (15). Vitamin D supplementation is being increasingly investigated in the context of chronic musculoskeletal pain and even peripheral neuropathic pain relief, and results so far suggest that these types of pain may be relieved by normalizing vitamin D levels $(16,17)$.

Vitamin D deficiency is a global health concern, being highly prevalent among various populations, and can be caused by reduced exposure to direct sunlight and/or reduced dietary intake (18). In the present study, it was hypothesized that musculoskeletal pain in patients with T2DM is inversely associated with serum 25-hydroxyvitamin D levels. The aims were i) to investigate the frequency of musculoskeletal pain in patients with T2DM according to body site; ii) to determine their serum 25-hydroxyvitamin D levels; iii) to assess their anxiety, depression and neuropathy scores; and iv) to investigate any association between pain measurements and serum 25-hydroxyvitamin D concentration, anxiety, depression and neuropathy scores.

\section{Materials and methods}

Participants. Patients with a confirmed diagnosis of T2DM were recruited from the King Abdullah University Hospital in Ramtha, Jordan between February and August 2016. Patients who had received vitamin D supplementation in the previous 3 months and patients with chronic renal failure and/or chronic liver disease were excluded from the study. All participants agreed to participate in the study by signing informed consent forms after discussing the study purpose and procedure. The study protocol received ethics approval by the Institutional Research Board of King Abdullah University Hospital and Jordan University of Science and Technology (Irbid, Jordan; approval no. 20150266).

Sample size. The number of study subjects was calculated based on other studies $(19,20)$ that determined the prevalence of vitamin D deficiency and insufficiency in patients with T2DM. In the Middle East, the prevalence of vitamin D deficiency and insufficiency in patients with T2DM between 2010 and 2013 ranged from 98.5\% in Saudi Arabia (19) to $89.7 \%$ in Iran (20). The following formula was used: Sample size $=(\mathrm{t})^{2}(\mathrm{p})(\mathrm{q}) / \mathrm{d}^{2}$, where $\mathrm{t}=1.96(\mathrm{t}$-value, $95 \%$ confidence interval), $\mathrm{p}=0.02-0.10$ [estimated prevalence of vitamin $\mathrm{D}$ deficiency and insufficiency based on other studies $(19,20)]$, $\mathrm{q}=1-\mathrm{p}$ and $\mathrm{d}=0.05$ (margin of error based on $95 \%$ confidence interval and 5\% error) (21). Therefore, the sample size based on the two abovementioned studies ranged between 18 and 142 patients. The present study included 124 participants, which represented a response rate of $89.21 \%$. Control subjects were not included due to the difficulty in recruiting a sufficient control population with normal vitamin D concentration from our Arab population, due to the high prevalence of vitamin D deficiency $(19,20)$. This was expected as most individuals do not expose sufficiently to sunlight because of the traditional dress of the region that covers most of the body.
Data collection. General information on patient age, sex, smoking status, vitamin D supplementation, use of statins and history of chronic renal failure and/or chronic liver disease were obtained from medical records and through self-reporting. Body weight was measured in $\mathrm{kg}$ using Detecto scales (Detecto Scale, Webb City, MO, USA) and height was measured in $\mathrm{cm}$ using a fixed metric scale in the clinic. BMI was calculated as body weight $(\mathrm{kg}) /$ height $(\mathrm{m})^{2}$.

Assessment of musculoskeletal pain. Musculoskeletal pain was assessed using two questions from the PainDETECT questionnaire (22). The first question asked participants to locate sites of usual pain by marking the area of pain on an illustration of human body. Then, the number of painful sites was calculated as a continuous variable for pain assessment. In addition, the number $(\%)$ of participants who were complaining of pain for each body site was reported. The second question asked participants to indicate average pain intensity over the last month using a scale from $0-10$ ( 0 , no pain; 10 , maximum pain).

Assessment of anxiety, depression and neuropathy. Anxiety and depression were assessed using an Arabic version of the Hospital Anxiety and Depression Scale (23). Neuropathy was assessed using an Arabic version of the PainDETECT questionnaire (22). Individuals with 0-12 neuropathy scores were considered as nociceptive, individuals with 13-18 neuropathy scores were considered as unclear and individuals with 19-38 neuropathy scores were considered as neuropathic. Both questionnaires were translated from English to Arabic using a standard forward-backward translation method.

Blood sampling and laboratory measurements. Venous blood samples $(10 \mathrm{ml})$ were collected following overnight fasting to measure fasting blood sugar (FBS), glycated hemoglobin (HbAlc) and 25-hydroxyvitamin D levels. Within $2 \mathrm{~h}$ of collection, serum was prepared by centrifuging the blood samples for $8 \mathrm{~min}$ at $2,100 \mathrm{x} \mathrm{g}$ at room temperature using a high-speed Jouan MR23i centrifuge (Thermo Fisher Scientific, Inc., Waltham, MA, USA). FBS was measured by the hexokinase method (24) using a Hitachi 902 auto-analyzer (Roche Diagnostics GmbH, Mannheim, Germany). HbA1c was measured by turbidimetric inhibition immunoassay (25) using the cobas b 101 system (Roche Diagnostics GmbH). The concentration of 25-hydroxyvitamin D was measured by liquid chromatography-tandem mass spectrometry (LC-MS/MS) using an API-3200 triple quadrupole mass spectrometer (Applied Biosystems; Thermo Fisher Scientific, Inc.) according to guidelines by the National Institute of Standards and Technology (NIST) as previously reported (26).

Statistical analysis. Statistical analysis was performed using IBM SPSS version 20 (IBM Corp., Armonk, NY, USA). Non-normally distributed continuous variables were log-transformed prior to analysis. Data for continuous variables are expressed as the mean \pm standard deviation or median (25th-75th percentiles). Descriptive data were expressed as frequency (\%). The $\chi^{2}$ and Fisher's exact tests were used to detect significant differences between categorical variables. One-way analysis of variance was used to detect significant differences in continuous variables between patients with 
(>30 ng/ml; n=16), insufficient (20-30 ng/ml; n=34) and deficient ( $<20 \mathrm{ng} / \mathrm{ml} ; \mathrm{n}=74)$ (26) vitamin $\mathrm{D}$ levels followed by Tukey's post hoc tests for multiple comparisons. The Pearson product-moment test was used to detect correlations between continuous variables and multiple linear regression analysis was performed to detect associations between pain measurements and other variables including neuropathy and anxiety scores, duration of DM, sex and statin therapy. All P-values were two-sided and considered statistically significant at $<0.05$.

\section{Results}

Characteristics of participants. Of 139 potential study subjects with T2DM, 124 (47 men and 77 women) agreed to participate in this study (response rate, 89.2\%). The participants' age ranged from 43 to 79 years, with a mean age of $59.3 \pm 9.5$ years. The duration of T2DM ranged from 1 to 17 years, with a mean of $5.0 \pm 3.4$ years. The mean BMI was $30.9 \pm 3.6 \mathrm{~kg} / \mathrm{m}^{2}$ and the median values of FBS and HbA1c were 7.7 (6.1-10.4) mmol/l and 7.5 (6.6-8.8)\%, respectively. A total of 21 (16.9\%) participants were current smokers, while $20(16.1 \%)$ and $83(66.9 \%)$ participants were former smokers and non-smokers, respectively.

Assessment of musculoskeletal pain. Table I illustrates the frequency of pain according to body location. The neck $(60.5 \%)$, lower back $(60.5 \%)$, head $(56.5 \%)$, right knee $(49.2 \%)$, right shoulder $(39.5 \%)$, left knee $(39.5 \%)$, right lower leg (39.5\%), left lower leg (34.7\%) and feet (33.9\%) were the most common painful sites reported by the study participants. The mean number of painful sites was $5.9 \pm 3.0$ and the mean pain intensity over the last month was 3.6 \pm 2.4 (data not shown).

Assessment of anxiety, depression and neuropathy scores. Abnormal anxiety scores were determined in $25(20.2 \%)$ participants, while $28(22.6 \%)$ and $71(57.3 \%)$ participants were considered to have borderline and normal anxiety scores, respectively. Abnormal depression scores were reported in $41(33.1 \%)$ participants, while 37 (29.8\%) and $46(37.1 \%)$ participants were considered to have borderline and normal depression scores, respectively. Neuropathy was indicated in 38 (30.6\%) participants, while $36(29.0 \%)$ and $50(40.3 \%)$ participants were considered to have unclear and normal neuropathy scores, respectively (data not shown).

Determination of study variables according to vitamin $D$ status. As presented in Table II, the number of painful sites, pain intensity over the last month, anxiety, depression and neuropathy scores, BMI and HbA1c were not significantly associated with vitamin D status (all $\mathrm{P}>0.05$ ). In addition, vitamin D status was not associated with sex or statin therapy, and there was no statistically significant difference in age, FBS or duration of DM among patients with sufficient, insufficient and deficient vitamin D levels (all P>0.05).

Association between pain measurements and study variables. As presented in Table III, both the number of painful sites and pain intensity over the last month were directly correlated with the duration of $\mathrm{DM}$ and neuropathy score (all $\mathrm{P}<0.05$ ).
Table I. Frequency of pain according to body site.

\begin{tabular}{lc}
\hline Site of pain & $\mathrm{n}(\%)$ \\
\hline Neck & $75(60.5)$ \\
Lower back & $75(60.5)$ \\
Head & $70(56.5)$ \\
Right knee & $61(49.2)$ \\
Right shoulder & $49(39.5)$ \\
Left knee & $49(39.5)$ \\
Right lower leg & $49(39.5)$ \\
Left lower leg & $43(34.7)$ \\
Feet & $42(33.9)$ \\
Right forearm & $33(26.6)$ \\
Left forearm & $32(25.8)$ \\
Left shoulder & $29(23.4)$ \\
Right hand & $28(22.6)$ \\
Left arm & $26(21.0)$ \\
Right arm & $25(20.2)$ \\
Left hand & $23(18.5)$ \\
Right upper leg & $16(12.9)$ \\
Left upper leg & $14(11.3)$ \\
&
\end{tabular}

Pain intensity over the last month was also directly correlated with the mean number of painful sites $(\mathrm{P}<0.01)$. The association of the mean number of painful sites with anxiety score was determined as not statistically significant $(\mathrm{P}=0.06)$. Furthermore, the pain measurements were not significantly correlated with 25-hydroxyvitamin D concentration, age, BMI, HbA1c, FBS or depression score (all P>0.05). Further multiple linear regression analyses (Table IV) identified the number of painful sites and pain intensity over the last month as dependent variables, both of which were significantly associated with neuropathy score $(\mathrm{P}<0.05)$. In addition, pain intensity over the last month was significantly associated with the mean number of painful sites $(\mathrm{P}<0.01)$. Neither the number of painful sites nor pain intensity over the last month was identified to be significantly associated with sex or the use of statins (all $\mathrm{P}>0.05$ ).

Correlation between vitamin D level and neuropathy score. As pain measurements were significantly associated with neuropathy score, further analysis was performed to determine whether there is an association between neuropathy score and 25-hydroxyvitamin D concentration. As depicted in Fig. 1, there was no significant correlation between 25-hydroxyvitamin $\mathrm{D}$ concentration and neuropathy score $(\mathrm{P}>0.05)$.

\section{Discussion}

The present study demonstrated that musculoskeletal pain was highly prevalent among patients with T2DM. Over $50 \%$ of the study participants suffered from chronic neck pain, lower back pain or headache. Similarly, Molsted et al (7) reported that 60 and $52 \%$ of their study participants with T2DM were suffering from lower back and neck pain, respectively. The pain frequency in other body sites was lower, and these results 
Table II. Determination of study variable associations according to vitamin D status.

\begin{tabular}{|c|c|c|c|c|}
\hline \multirow[b]{2}{*}{ Variable } & \multicolumn{3}{|c|}{ 25-hydroxyvitamin D status } & \multirow[b]{2}{*}{ P-value } \\
\hline & $\begin{array}{c}\text { Sufficient, } \\
>30 \mathrm{ng} / \mathrm{ml}(\mathrm{n}=16)\end{array}$ & $\begin{array}{c}\text { Insufficient, } \\
20-30 \mathrm{ng} / \mathrm{ml}(\mathrm{n}=34)\end{array}$ & $\begin{array}{c}\text { Deficient, } \\
<20 \mathrm{ng} / \mathrm{ml}(\mathrm{n}=74)\end{array}$ & \\
\hline Age, years & $58.0 \pm 10.2$ & $58.6 \pm 8.6$ & $59.3 \pm 9.5$ & 0.76 \\
\hline Sex & & & & 0.20 \\
\hline Male & $3(18.8)$ & $15(44.1)$ & $29(39.2)$ & \\
\hline Female & $13(81.2)$ & $19(55.9)$ & $45(60.8)$ & \\
\hline BMI $\left(\mathrm{kg} / \mathrm{m}^{2}\right)$ & & & & 0.66 \\
\hline Normal (18.6-24.9) & $1(6.2)$ & $0(0.0)$ & $4(5.4)$ & \\
\hline Overweight (25-29.9) & $7(43.8)$ & $15(44.1)$ & $28(37.8)$ & \\
\hline Obese $(>30)$ & $8(50.0)$ & $19(55.9)$ & $42(56.8)$ & \\
\hline Duration of diabetes mellitus, years & $4.9 \pm 4.1$ & $4.7 \pm 2.6$ & $5.2 \pm 3.5$ & 0.80 \\
\hline $\mathrm{HbA1c}$ & & & & 0.69 \\
\hline$<7 \%$ (controlled) & $7(43.8)$ & $14(41.2)$ & $25(33.8)$ & \\
\hline$\geq 7 \%$ (uncontrolled) & $9(56.2)$ & $20(58.8)$ & $49(66.2)$ & \\
\hline Log[fasting blood sugar $(\mathrm{mmol} / \mathrm{l})]$ & $0.9 \pm 0.1$ & $0.9 \pm 0.2$ & $0.9 \pm 0.2$ & 0.35 \\
\hline Smoking status & & & & 0.92 \\
\hline Current smoker & $2(12.5)$ & $6(17.6)$ & $13(17.6)$ & \\
\hline Former smoker & $2(12.5)$ & $7(20.6)$ & $11(14.9)$ & \\
\hline None smoker & $12(75.0)$ & $21(61.8)$ & $50(67.7)$ & \\
\hline Number of painful sites & $4.69 \pm 3.0$ & $6.7 \pm 3.8$ & $5.8 \pm 2.6$ & 0.09 \\
\hline Pain intensity over the last month (0-10 scale) & $3.8 \pm 2.7$ & $4.2 \pm 2.6$ & $3.4 \pm 2.2$ & 0.24 \\
\hline Anxiety score & & & & 0.18 \\
\hline Normal (0-7) & $5(31.2)$ & $21(61.8)$ & $45(60.8)$ & \\
\hline Borderline (8-10) & $5(31.2)$ & $6(17.6)$ & $17(23.0)$ & \\
\hline Abnormal (11-21) & $6(37.5)$ & $7(20.6)$ & $12(16.2)$ & \\
\hline Depression score & & & & 0.40 \\
\hline Normal (0-7) & $6(37.5)$ & $11(32.4)$ & $29(39.2)$ & \\
\hline Borderline (8-10) & $2(12.5)$ & $11(32.4)$ & $24(32.4)$ & \\
\hline Abnormal (11-21) & $8(50.0)$ & $12(35.3)$ & $21(28.4)$ & \\
\hline Neuropathy score & & & & 0.68 \\
\hline Nociceptive (0-12) & $6(37.5)$ & $18(38.2)$ & $31(41.9)$ & \\
\hline Unclear (13-18) & $4(25.0)$ & $18(38.2)$ & $19(25.7)$ & \\
\hline Neuropathic (19-38) & $6(37.5)$ & $8(23.5)$ & $24(32.4)$ & \\
\hline Statin therapy & & & & 0.15 \\
\hline Yes & $7(43.8)$ & $16(47.1)$ & $47(63.5)$ & \\
\hline No & $9(56.2)$ & $18(52.9)$ & $27(36.5)$ & \\
\hline
\end{tabular}

P-values were determined by $\chi^{2}$ or Fisher's exact tests for categorical variables and one-way analysis of variance test for continuous variables. Data are presented as frequency $(\%)$ or mean \pm standard deviation. Vitamin D reference ranges were as defined by Sadat-Ali et al (26) in their study based on the same method of vitamin D determination. Anxiety, depression and neuropathy score reference ranges were defined as in the PainDETECT and Hospital Anxiety and Depression Scale scoring systems $(22,23)$. Reference ranges for BMI were as defined by Nuttall $(33)$. HbA1c reference levels were as defined by Esposito et al (34). BMI, body mass index; HbA1c, glycated hemoglobin.

are comparable with those reported by other studies, including that by Kidwai et al (27), in which the pain frequencies in the hands, shoulders and upper limbs were 20.5, 19.5 and $32.9 \%$, respectively. In the present study, pain in the lower extremities, including the knees, lower legs and feet, was more common compared with pain in the upper extremities (the arms, forearms, hands, left shoulder and upper legs). This suggests that the lower extremities, which bear the weight of the body, are more susceptible to musculoskeletal pain, particularly when the subjects have increased BMI (96\% of the current study participants were overweight or obese). This was also confirmed by Viester et al (8), who reported that increased BMI was associated with the development of musculoskeletal pain in the lower extremities. 
Table III. Correlation between pain measurements and other variables.

\begin{tabular}{|c|c|c|c|c|}
\hline \multirow[b]{2}{*}{ Variable } & \multicolumn{2}{|c|}{ Number of painful sites } & \multicolumn{2}{|c|}{ Pain intensity over the last month } \\
\hline & $\mathrm{r}$ & $\mathrm{P}$-value & $\mathrm{r}$ & P-value \\
\hline Age, years & 0.09 & 0.33 & 0.01 & 0.27 \\
\hline Body mass index, $\mathrm{kg} / \mathrm{m}^{2}$ & 0.14 & 0.13 & 0.14 & 0.13 \\
\hline Log[glycated hemoglobin (\%)] & -0.11 & 0.23 & $<-0.01$ & 0.99 \\
\hline $\log [$ fasting blood sugar $(\mathrm{mmol} / \mathrm{l})]$ & -0.06 & 0.54 & 0.03 & 0.78 \\
\hline Duration of diabetes mellitus, years & 0.19 & 0.03 & 0.20 & $\mathbf{0 . 0 3}$ \\
\hline Log[25-hydroxyvitamin D (ng/ml)] & $<-0.01$ & 0.96 & 0.05 & 0.61 \\
\hline Anxiety score & 0.17 & 0.06 & 0.15 & 0.11 \\
\hline Depression score & 0.02 & 0.87 & 0.07 & 0.42 \\
\hline Neuropathy score & 0.63 & 0.00 & 0.46 & 0.00 \\
\hline Number of painful sites & - & - & 0.46 & 0.00 \\
\hline
\end{tabular}

P-values were determined by Pearson product-moment correlation test. Statistically significant values $(\mathrm{P}<0.05)$ are emboldened. $\mathrm{r}$, correlation coefficient.

Table IV. Association between pain measurements and other variables.

\begin{tabular}{|c|c|c|c|c|c|c|c|}
\hline Dependent variable & $\mathrm{R}^{2}$ & Analysis of variance & Model & B & $\beta$ & t-value & P-value \\
\hline \multirow{6}{*}{$\begin{array}{l}\text { Pain intensity } \\
\text { over the last month }\end{array}$} & \multirow[t]{6}{*}{0.28} & \multirow[t]{6}{*}{$\mathrm{F}=9.17, \mathrm{P}<0.01$} & Constant & 0.47 & - & 0.61 & 0.54 \\
\hline & & & Neuropathy score & 0.08 & 0.24 & 2.43 & 0.02 \\
\hline & & & Duration of DM & 0.08 & 0.11 & 1.30 & 0.20 \\
\hline & & & Number of painful sites & 0.22 & 0.28 & 2.90 & $<0.01$ \\
\hline & & & Sex & 0.38 & 0.08 & 0.95 & 0.34 \\
\hline & & & Statin therapy & -0.31 & -0.07 & -0.80 & 0.43 \\
\hline \multirow{6}{*}{$\begin{array}{l}\text { Number of } \\
\text { painful sites }\end{array}$} & \multirow[t]{6}{*}{0.36} & \multirow[t]{6}{*}{$\mathrm{F}=13.27, \mathrm{P}<0.01$} & Constant & 2.69 & - & 2.72 & 0.01 \\
\hline & & & Neuropathy score & 0.24 & 0.56 & 6.87 & $<0.01$ \\
\hline & & & Duration of DM & 0.07 & 0.08 & 0.97 & 0.33 \\
\hline & & & Anxiety score & 0.03 & 0.04 & 0.56 & 0.58 \\
\hline & & & Sex & -0.04 & -0.01 & -0.09 & 0.93 \\
\hline & & & Statin therapy & -0.47 & -0.08 & -1.00 & 0.32 \\
\hline
\end{tabular}

P-values were determined by multiple linear regression analysis. Statistically significant values $(\mathrm{P}<0.05)$ are emboldened. $\mathrm{R}^{2}$, squared coefficient of determination; B, unstandardized coefficient; $\beta$, standardized coefficient; F, F-statistic; DM, diabetes mellitus.

The major goal of the present study was to investigate whether musculoskeletal pain in patients with T2DM is associated with serum 25 -hydroxyvitamin D levels. The results identified no significant difference in the number of painful sites or pain intensity over the last month among patients with sufficient, insufficient or deficient 25-hydroxyvitamin D levels. In addition, there was no significant correlation between serum 25-hydroxyvitamin D concentration and the number of painful sites or pain intensity over the last month. Accordingly, measures of musculoskeletal pain in T2DM were expected to have no association with serum 25-hydroxyvitamin D levels. However, the results of the present study support the findings of Shipton and Shipton (28), who reported that the scientific evidence for using vitamin $\mathrm{D}$ to treat chronic pain is limited due to the lack of supporting randomized controlled trials. As musculoskeletal pain may result from anxiety and depression (15), which may also be caused by vitamin D deficiency $(13,14)$, it was investigated whether there was any correlation of pain measurements with anxiety and depression scores. However, the results revealed no significant correlation between pain measurements and anxiety or depression scores. Furthermore, anxiety and depression scores were not significantly correlated with serum 25-hydroxyvitamin $\mathrm{D}$ levels. These results indicate that musculoskeletal pain in T2DM patients was not significantly associated with anxiety or depression scores.

By contrast, both the number of painful sites and pain intensity over the last month were directly correlated with the duration of T2DM and neuropathy score, indicating that musculoskeletal pain increases with prolonged duration of T2DM and increase in neuropathy score. Further multiple linear regression analyses demonstrated that the number of 


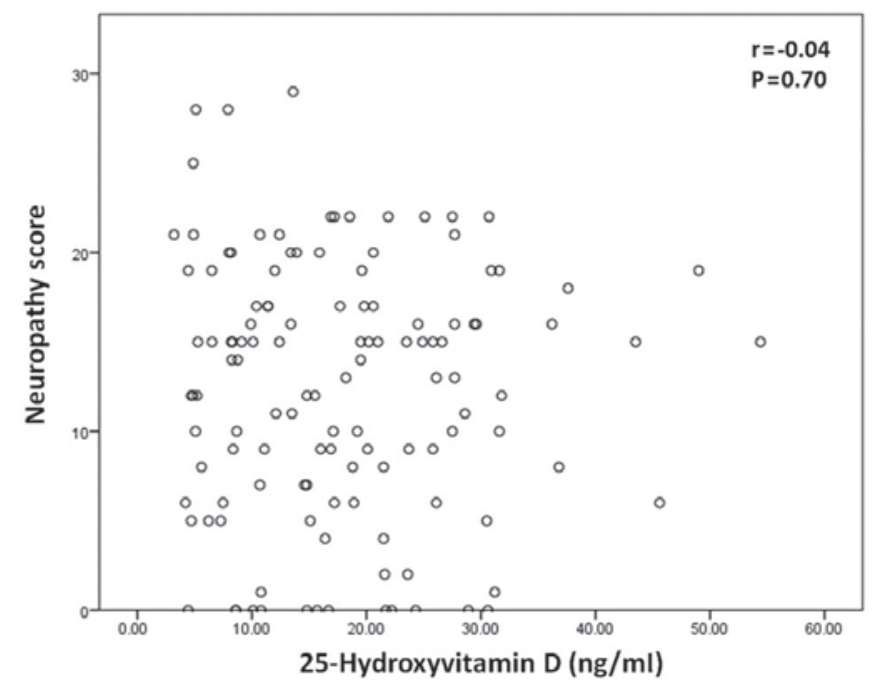

Figure 1. Scatter plot of 25-hydroxyvitamin D plasma concentration vs. neuropathy score in patients with type 2 diabetes mellitus.

painful sites and pain intensity over the last month may be predicted from the neuropathy score, suggesting that these pain measurements are associated with the neuropathy score. Further correlation analysis revealed no statistically significant correlation between neuropathy score and vitamin D level. Meanwhile, pain intensity over the last month was associated with the number of painful sites, indicating that pain intensity may also be predicted from the number of painful sites.

Musculoskeletal pain may be caused by statin therapy as an adverse outcome that affects $5-18 \%$ of patients treated with these lipid-lowering agents (29). Morioka et al (30) reported that statin users with vitamin D deficiency complained of musculoskeletal pain at twice the frequency of individuals who were not taking statins. In addition, they concluded that vitamin $\mathrm{D}$ deficiency modifies the risk of musculoskeletal pain in statin users (30). In the present study, it was also investigated whether there are associations between statin therapy use, vitamin D deficiency and the development of musculoskeletal pain in patients with T2DM. Statin therapy was reported in $43.5 \%$ of the participants. Vitamin D status was not significantly correlated with the use of statins; pain measurements, including both pain intensity over the last month and number of painful sites, were also not significantly associated with statin therapy.

Collectively, the findings of the present study demonstrated that musculoskeletal pain was prevalent among patients with T2DM, and that pain measurements were not associated with serum 25-hydroxyvitamin D levels, despite accumulating evidence supports vitamin D supplementation as a treatment option for such manifestations $(17,31,32)$. Measures of musculoskeletal pain were significantly correlated with the duration of T2DM and neuropathy score, and these measures may be predicted from the neuropathy score. Thus, preventing and treating diabetic neuropathy should be prioritized as a treatment option for managing musculoskeletal pain in patients with T2DM. However, the significance of these findings may be limited by the cross-sectional design of the present study. A follow-up study is required to assess whether vitamin $\mathrm{D}$ supplementation or neuropathy treatment may improve musculoskeletal pain in patients with T2DM. Another limitation is that self-reporting was used to assess pain, neuropathy, anxiety and depression among patients. Funding limitations prevented consultation of a neurology clinic to definitively diagnose neuropathy, anxiety and depression according to the clinical guidelines; however, the questionnaires $(22,23)$ that were used to assess these variables are well-validated and considered reasonably reliable for research purposes. In addition, funding limitations prevented enrollment of a larger sample size, which may affect applications of the findings to a general population. However, despite the limitations of the present study, the current findings may draw attention toward the assessment of musculoskeletal manifestations in patients with T2DM, as well as encourage further investigations to assess the etiology of musculoskeletal pain in patients with T2DM and determine whether vitamin D supplementation and management of neuropathy may be used for pain relief in such patients.

\section{Acknowledgements}

Not applicable.

\section{Funding}

The present study was supported by the Deanship of Research at Jordan University of Science and Technology (grant no. 2015/266).

\section{Availability of data and materials}

The datasets used and/or analyzed during the current study are available from the corresponding author on reasonable request.

\section{Authors' contributions}

MJA was responsible for study design, data analysis and manuscript writing. KKAR was responsible for results interpretation and manuscript editing. LQK was responsible for data collection and patient recruitment. NAS was responsible for patient recruitment and diagnosis. All authors read and approved the final manuscript.

\section{Ethics approval and consent to participate}

The present study received ethics approval from the Institutional Research Board of King Abdullah University Hospital and Jordan University of Science and Technology, Irbid, Jordan (approval no. 20150266). All participants agreed to participate in the study by signing informed consent forms after discussing the study purpose and procedure.

\section{Consent for publication}

All participants provided written informed consent permitting publication of relevant data following anonymization of personal information.

\section{Competing interests}

The authors declare that they have no competing interests. 


\section{References}

1. American Diabetes Association: Diagnosis and classification of diabetes mellitus. Diabetes Care 33 (Suppl 1): 62-69, 2010.

2. No authors listed: Pharmacologic management of type 2 diabetes: 2016 interim update. Can J Diabetes 40: 193-195, 2016.

3. Zhu H, Zhu Y and Leung SW: Is self-monitoring of blood glucose effective in improving glycaemic control in type 2 diabetes without insulin treatment: A meta-analysis of randomised controlled trials. BMJ Open 6: e010524, 2016.

4. Stolar M: Glycemic control and complications in type 2 diabetes mellitus. Am J Med 123 (Suppl): S3-S11, 2010.

5. Singh VP, Bali A, Singh N and Jaggi AS: Advanced glycation end products and diabetic complications. Korean J Physiol Pharmacol 18: 1-14, 2014.

6. Pai LW, Hung CT, Li SF, Chen LL, Chung Y and Liu HL: Musculoskeletal pain in people with and without type 2 diabetes in Taiwan: A population-based, retrospective cohort study. BMC Musculoskelet Disord 16: 364, 2015.

7. Molsted S, Tribler J and Snorgaard O: Musculoskeletal pain in patients with type 2 diabetes. Diabetes Res Clin Pract 96: 135-140, 2012.

8. Viester L, Verhagen EA, Oude Hengel KM, Koppes LL, van der Beek AJ and Bongers PM: The relation between body mass index and musculoskeletal symptoms in the working population. BMC Musculoskeletal Disorders 14: 238, 2013.

9. Marchettini P, Lacerenza M, Mauri E and Marangoni C: Painful peripheral neuropathies. Curr Neuropharmacol 4: 175-181, 2006

10. Straube S, Andrew Moore R, Derry S and McQuay HJ: Vitamin D and chronic pain. Pain 141: 10-13, 2009.

11. Wasserman RH: Intestinal absorption of calcium and phosphorus. Fed Proc 40: 68-72, 1981.

12. Lips P and van Schoor NM: The effect of vitamin D on bone and osteoporosis. Best Pract Res Clin Endocrinol Metab 25: 585-591, 2011.

13. Hoang MT, Defina LF, Willis BL, Leonard DS, Weiner MF and Brown ES: Association between low serum 25-hydroxyvitamin D and depression in a large sample of healthy adults: The Cooper Center longitudinal study. Mayo Clin Proc 86: 1050-1055, 2011.

14. Armstrong DJ, Meenagh GK, Bickle I, Lee AS, Curran ES and Finch MB: Vitamin D deficiency is associated with anxiety and depression in fibromyalgia. Clin Rheumatol 26: 551-554, 2007.

15. de Heer EW, Gerrits MM, Beekman AT, Dekker J, van Marwijk HW, de Waal MW, Spinhoven P, Penninx BW, and van der Feltz-Cornelis CM: The association of depression and anxiety with pain: A study from NESDA. PLoS One 9: e106907, 2014.

16. Bell DS: Reversal of the symptoms of diabetic neuropathy through correction of vitamin D deficiency in a type 1 diabetic patient. Case Rep Endocrinol 2012: 165056, 2012.

17. Kragstrup TW: Vitamin D supplementation for patients with chronic pain. Scand J Prim Health Care 29: 4-5, 2011.

18. Palacios C and Gonzalez L: Is vitamin D deficiency a major global public health problem? J Steroid Biochem Mol Biol 144 Pt A: $138-145,2014$.
19. Alhumaidi M, Agha A and Dewish M: Vitamin D deficiency in patients with type-2 diabetes mellitus in southern region of saudi arabia. Maedica (Buchar) 8: 231-236, 2013.

20. Bayani MA, Akbari R, Banasaz B and Saeedi F: Status of vitamin-D in diabetic patients. Caspian J Intern Med 5: 40-42, 2014.

21. Higgins JEBWKC: Organizational research: Determining appropriate sample size in survey research. Inf Technol Learn Perform J 19: 43-50, 2001.

22. Freynhagen R, Baron R, Gockel U and Tölle TR: painDETECT: A new screening questionnaire to identify neuropathic components in patients with back pain. Curr Med Res Opin 22: 1911-1920, 2006.

23. Zigmond AS and Snaith RP: The hospital anxiety and depression scale. Acta Psychiatr Scand 67: 361-370, 1983.

24. Schmidt FH: Blood glucose levels in capilary blood of adults assessed by the hexokinase method (author's transl). Klin Wochenschr 51: 520-522, 1973 (In German).

25. Genc S, Omer B, Aycan-Ustyol E, Ince N, Bal F and Gurdol F: Evaluation of turbidimetric inhibition immunoassay (TINIA) and HPLC methods for glycated haemoglobin determination. J Clin Lab Anal 26: 481-485, 2012.

26. Sadat-Ali M, Al-Elq AH, Al-Shaikh IH, Al-Turki HA, Al-Ali AK and Al-Othman AA: Assessment of low vitamin D among Saudi Arabians. Did we overshoot the runway? Saudi Med J 35: 1243-1249, 2014.

27. Kidwai SS, Wahid L, Siddiqi SA, Khan RM, Ghauri I, Sheikh I: Upper limb musculoskeletal abnormalities in type 2 diabetic patients in low socioeconomic strata in Pakistan. BMC Res Notes 6: 16, 2013.

28. Shipton EE and Shipton EA: Vitamin D deficiency and pain: Clinical evidence of low levels of vitamin D and supplementation in chronic pain states. Pain Ther 4: 67-87, 2015.

29. Di Stasi SL, MacLeod TD, Winters JD and Binder-Macleod SA: Effects of statins on skeletal muscle: A perspective for physical therapists. Phys Ther 90: 1530-1542, 2010.

30. Morioka TY, Lee AJ, Bertisch S and Buettner C: Vitamin D status modifies the association between statin use and musculoskeletal pain: a population based study. Atherosclerosis 238: 77-82, 2015.

31. Le Goaziou MF, Bodier E, Souweine G, Moreau A, Perdrix C, Flori $\mathrm{M}$ and Dupraz C: Musculoskeletal chronic pains and vitamin D deficiency. Study before after in general practice. Presse Med 42 (4 Pt 1): e106-113, 2013 (In French).

32. Gendelman O, Itzhaki D, Makarov S, Bennun M and Amital $\mathrm{H}$ : A randomized double-blind placebo-controlled study adding high dose vitamin D to analgesic regimens in patients with musculoskeletal pain. Lupus 24: 483-489, 2015.

33. Nuttall FQ: Body mass index: Obesity, BMI, and health: A critical review. Nutr Today 50: 117-128, 2015.

34. Esposito K, Chiodini P, Bellastella G, Maiorino MI and Giugliano D: Proportion of patients at HbAlc target $<7 \%$ with eight classes of antidiabetic drugs in type 2 diabetes: Systematic review of 218 randomized controlled trials with 78945 patients. Diabetes Obes Metab 14: 228-233, 2012. 